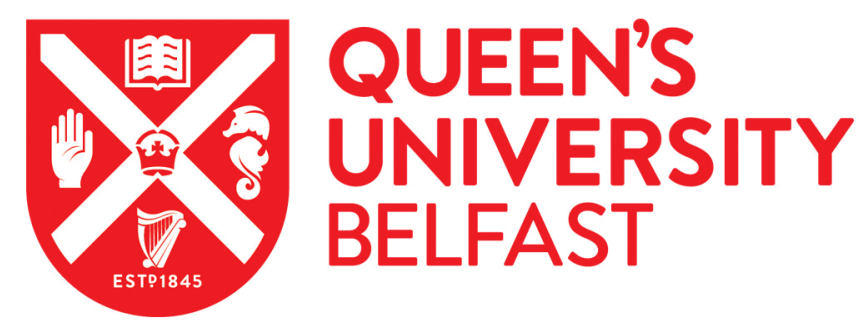

\title{
Role of information backflow in the emergence of quantum Darwinism
}

Milazzo, N., Lorenzo, S., Paternostro, M., \& Palma, G. M. (2019). Role of information backflow in the emergence of quantum Darwinism. Physical Review A, 100(1), [012101]. https://doi.org/10.1103/PhysRevA.100.012101

\author{
Published in: \\ Physical Review A
}

Document Version:

Publisher's PDF, also known as Version of record

Queen's University Belfast - Research Portal:

Link to publication record in Queen's University Belfast Research Portal

\section{Publisher rights}

(C2019 American Physical Society.

This work is made available online in accordance with the publisher's policies. Please refer to any applicable terms of use of the publisher.

\section{General rights}

Copyright for the publications made accessible via the Queen's University Belfast Research Portal is retained by the author(s) and / or other copyright owners and it is a condition of accessing these publications that users recognise and abide by the legal requirements associated with these rights.

Take down policy

The Research Portal is Queen's institutional repository that provides access to Queen's research output. Every effort has been made to ensure that content in the Research Portal does not infringe any person's rights, or applicable UK laws. If you discover content in the Research Portal that you believe breaches copyright or violates any law, please contact openaccess@qub.ac.uk. 


\title{
Role of information backflow in the emergence of quantum Darwinism
}

\author{
Nadia Milazzo, ${ }^{1,2}$ Salvatore Lorenzo, ${ }^{3}$ Mauro Paternostro, ${ }^{4}$ and G. Massimo Palma ${ }^{5}$ \\ ${ }^{1}$ Institut für theoretische Physik, Universität Tübingen, 72076 Tübingen, Germany \\ ${ }^{2}$ LPTMS, UMR 8626, CNRS, Univ. Paris-Sud, Université Paris-Saclay, 91405 Orsay, France \\ ${ }^{3}$ Dipartimento di Fisica e Chimica, Università degli Studi di Palermo, via Archirafi 36, I-90123 Palermo, Italy \\ ${ }^{4}$ Centre for Theoretical Atomic, Molecular, and Optical Physics, School of Mathematics and Physics, Queen's University, \\ Belfast BT7 1NN, United Kingdom \\ ${ }^{5}$ NEST, Istituto Nanoscienze-CNR and Dipartimento di Fisica e Chimica, Università degli Studi di Palermo, via Archirafi 36, I-90123 \\ Palermo, Italy
}

(Received 18 February 2019; published 1 July 2019)

\begin{abstract}
Quantum Darwinism attempts to explain the emergence of objective reality of the state of a quantum system in terms of redundant information about the system acquired by independent noninteracting fragments of the environment. The consideration of interacting environmental elements gives rise to a rich phenomenology, including the occurrence of non-Markovian features, whose effects on objectification in the manner of quantum Darwinism needs to be fully understood. We study a model of local interaction between a simple quantum system and a multimode environment that allows for a clear investigation of the interplay between information trapping and propagation in the environment and the emergence of quantum Darwinism. We provide strong evidence of the correlation between non-Markovianity and quantum Darwinism in such a model, thus providing strong evidence of a potential link between such fundamental phenomena.
\end{abstract}

DOI: 10.1103/PhysRevA.100.012101

\section{INTRODUCTION}

The quantum Darwinism paradigm is one of the most recent and convincing attempts to explain the emergence of objective reality out of superpositions of quantum states (for a review see [1]). In this framework, the first key mechanism responsible for the transition from quantum to classical is the coupling of the system with an environment which acquires information about the state of the system with respect to the so-called pointer states, namely the eigenstates of the observable which is coupled with the environment [2-4]. If the system is in a specific pointer state it is left undisturbed by such coupling; if, however, the system is in a coherent superposition of pointer states it gets entangled with the environment. An external observer who can access the environment can therefore acquire information on the state of the system, leading to its objective existence.

The second key ingredient at the basis of quantum Darwinism is the particular structure of the environmental states which get entangled with the pointer state. The basic idea is that in a real scenario the environmental degrees of freedom are not traced out but rather accessed by different observers. The assumption is that information on the state of the system is redundantly encoded in multiple, independent fragments of the environment, which we assume to consist of a large set of noninteracting units. External observers can read the information on the system contained in separate, locally accessible fragments of the environment, with each fragment containing the same information on the system, and this leading to objective reality of the system state [5-7]. This is what naturally happens when an initial coherent superposition of system pointer states $|\Psi\rangle_{S}=\sum_{k=1}^{n} \psi_{k}\left|\pi_{k}\right\rangle_{S}$ evolves into a joint system-environment state with a branching structure

$$
\left|\Psi_{S E}\right\rangle=\sum_{k=1}^{n} \psi_{k}\left|\pi_{k}\right\rangle_{S} \bigotimes_{j=1}^{M}\left|\eta_{k}\right\rangle_{j},
$$

where the information about the system state $\left|\pi_{k}\right\rangle_{S}$ is imprinted into multiple copies of environmental states $\left|\eta_{k}\right\rangle$, thus becoming accessible to individual, distinct observers, that access separate fragments of the environment.

The phenomenology of quantum Darwinism has attracted a robust body of work, recently [8-21], while the first attempts at its experimental assessment have been reported [22-25]. Yet, the fundamental mechanism for its emergence and the features that characterize it are yet to be fully understood. On one hand, the relation with interesting alternative formulations for the emergence of objective reality through the formalism of quantum spectrum broadcasting structures needs clarifying [26-28]. On the other hand, it appears that quantum correlations have a significant influence on the qualification of quantum Darwinism [29]. Such interplay deserves a complete understanding in light of the relevance that, say, quantum entanglement has for the characterization of the quantum-toclassical transition. Finally, and most relevantly for the work reported in this paper, the possible influence that memory effects in the open-system dynamics of a quantum information carrier have on the emergence of objective reality has been the focus of controversy. While recent studies have suggested the detrimental role of non-Markovianity for the manifestation of quantum Darwinism [19,20], the relation between objectification and spectrum broadcasting structures appears to be loose [30]. 
A full characterization of quantum information flow between system end environment plays a crucial role not only in the understanding of the emergence of objective reality and of quantum Darwinism but also in the current quantitative description of quantum non-Markovianity. In recent years quantum information theory has provided new mathematical tools to better define quantum non-Markovian dynamics. In particular, a number of theoretical measures of the degree of quantum non-Markovianity of an open dynamics have been put forward [31-37]. Such measures have been used to identify the regions in the parameter space corresponding to Markovian and non-Markovian dynamics for a variety of environmental models [38-46]. All such measures capture the idea that non-Markovianity is linked to a backflow of quantum information from the environment to the system.

Such a link could be key in understanding the process of objectification that is at the core of Darwinism. In this paper we contribute to such an understanding by exploring the links between information backflow and the emergence of quantum Darwinism in a physically relevant scenario that is rather different from the configurations addressed so far. We consider a two-level system locally coupled to a single harmonic oscillator that is part of a one-dimensional interacting harmonic lattice that embodies the environment. This is distinct from the typical assumption of a system being collectively coupled to the elements of the environment $[19,20]$. Such a difference is not to be underestimated: in this situation, in fact, we expect information on the state of the system not to be copied onto separate fragments of the environment. Rather, quantum information about the system would flow through the environmental fragments via their mutual interaction. We show that when the system shows a Markovian evolution it shows also a Darwinistic behavior, while Darwinism disappears when the system dynamics is non-Markovian. We also explore how both effects are linked with the directionality of quantum information flow from the system to the environment.

The remainder of the paper is structured as follows. In Sec. II we introduce our model, solve the reduced dynamics of the system of interest, which turns out to be a time-dependent dephasing one, and quantify the corresponding degree of nonMarkovianity of such dynamics. Section III is devoted to the phenomenology of quantum Darwinism as non-Markovian effects settle into the dynamics of the system. In particular, in Sec. IV we link such phenomenology to the features of information flow across the environmental lattice, thus providing a clear physical assessment for the onset of Darwinism and non-Markovianity. Section V reports our conclusions.

\section{MODEL AND ITS DEGREE OF NON-MARKOVIANITY}

We consider an exactly soluble model in which we drop the assumption of independent subenvironments. Specifically, we consider an environment $\mathcal{E}$ consisting of a one-dimensional array of $N$ linearly coupled harmonic oscillators. On the other hand, the system $S$ is embodied by a two-level system that is locally coupled to one such oscillator (cf. Fig. 1). The Hamiltonian of the model (written in units such that $\hbar=1$ )

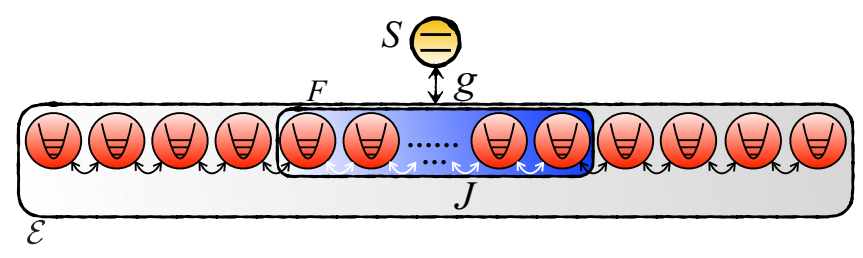

FIG. 1. Single two-level $S$ is locally coupled (at a strength $g$ ) to a harmonic oscillator that is part of a linear array of interacting oscillators $\mathcal{E}$. The interoscillator coupling rate is $J$. We study the mutual information that increasingly large fragments $F$ of the array share with system $S$. The fragments $F$ will all consist of sections centered at the qubit position.

reads

$$
\begin{aligned}
\hat{H}= & \frac{\omega_{0}}{2} \hat{\sigma}_{z}^{S}+\omega \sum_{j} \hat{a}_{j}^{\dagger} \hat{a}_{j} \\
& +J \sum_{j}\left(\hat{a}_{j}^{\dagger} \hat{a}_{j+1}+\text { H.c. }\right)+g \sigma_{z}\left(\hat{a}_{0}^{\dagger}+\hat{a}_{0}\right),
\end{aligned}
$$

where $\omega_{0}$ is the energy splitting between the states of $S, \hat{\sigma}_{z}^{S}$ is its $z$-Pauli matrix, $\omega$ is the frequency of the $j$ th local oscillators with bosonic creation (annihilation) operator $a_{j}^{\dagger}\left(a_{j}\right)$, and $j \in\{-(N-1) / 2,(N-1) / 2\}$. The bosonic intraenvironment coupling rate is $J$. We assume that $S$ is locally coupled at a rate $g$ to the central oscillator, which has label $j=0$.

Due to the coupling with the central harmonic oscillator, the information about the state of $S$ propagates along the environment $\mathcal{E}$, whose sections act as interacting environmental fragments which can be individually accessed. With such geometry, the way in which the environmental fragments acquire information about the state of the system is radically different from the noninteracting or the star-shaped scenario: quantum information must now propagate along the array and is not acquired simultaneously by the environmental fragments as in the case of a star geometry where the system is simultaneously coupled to $N$ (possibly interacting) subenvironments. We will show how the efficiency with which quantum information flows along the array affects the emergence of quantum Darwinism and determines non-Markovian effects in the reduced dynamics of $S$. In particular, we will show that the threshold in our parameter space at which we have an onset of nonMarkovianity is exactly the same as the one at which quantum Darwinism breaks down.

The key feature of our choice of environmental model is the possibility to switch from a local-oscillator picture to one based on normal modes. The exactly solvable nature of the environmental Hamiltonian also simplifies the establishment of a clear relationship between the information that is locally accessible and the information flux across $\mathcal{E}$.

In terms of normal modes $\hat{b}_{k}$ of the environment, the Hamiltonian reads

$$
\hat{H}=\frac{\omega_{0}}{2} \hat{\sigma}_{z}^{S}+\sum_{k} \Omega_{k} \hat{b}_{k}^{\dagger} \hat{b}_{k}+\frac{g}{\sqrt{N}} \hat{\sigma}_{z} \sum_{k}\left(\hat{b}_{k}^{\dagger}+\hat{b}_{k}\right),
$$

where $\hat{b}_{k}^{\dagger}=\sum_{j} e^{i k j} \hat{a}_{j}^{\dagger} / \sqrt{N}$ is the creation operator of mode $k=2 \pi n / N\left(n=-\frac{N-1}{2}, \ldots, \frac{N-1}{2}\right)$ and the frequency $\Omega_{k}$ of the $k$ th normal mode is determined by the dispersion relation 
$\Omega_{k}=\omega+\omega_{k}=\omega+2 J \cos k$. In the interaction picture with respect to the free-energy terms $\frac{\omega_{0}}{2} \hat{\sigma}_{z}^{S}+\sum_{k} \Omega_{k} \hat{b}_{k}^{\dagger} \hat{b}_{k}$, the time evolution operator takes the form of a collection of conditional displacement operators, one per normal mode, dependent on the state of the two-level system [47]. Explicitly, we have

$$
\hat{\mathcal{U}}_{I}(t)=e^{-i \phi} e^{-i \sigma_{z} \sum_{k}\left[\beta_{k}(t) \hat{b}_{k}^{\dagger}-\beta_{k}^{*}(t) \hat{b}_{k}\right]},
$$

where $\phi$ is an irrelevant global phase factor (arising from time ordering) and $\beta_{k}(t)=(g / \sqrt{N})\left(1-e^{i \Omega_{k} t}\right) / \Omega_{k}$ [48]. Such a propagator leads to decoherence, with the system states $\left\{|0\rangle_{S},|1\rangle_{S}\right\}$ as pointer states [2]. A linear superposition of the system's states such as $c_{0}|0\rangle_{S}+c_{1}|1\rangle_{S}$-with the environmental modes prepared in their vacuum state-evolves into the entangled state

$$
|\psi(t)\rangle_{S \mathcal{E}}=c_{0}|0\rangle_{S} \bigotimes_{k}\left|-\beta_{k}(t)\right\rangle+c_{1}|1\rangle_{S} \bigotimes_{k}\left|\beta_{k}(t)\right\rangle,
$$

where $\left|\beta_{k}(t)\right\rangle$ is a coherent state of mode $k$. Such dynamics leads to decoherence of the reduced density operator $\rho_{S}(t)$ of the system, with a decoherence function $e^{-\Gamma(t)}=\prod_{k}\left\langle\beta_{k}(t)\right|-$ $\left.\beta_{k}(t)\right\rangle$. An explicit calculation leads to [47]

$$
\Gamma(t)=\frac{4 g^{2}}{N} \sum_{k} \frac{1-\cos \Omega_{k} t}{\Omega_{k}^{2}} .
$$

Such reduced dynamics can be ascribed to the time-local master equation [33]

$$
\dot{\rho}_{S}(t)=\gamma(t)\left[\sigma_{z} \rho_{S}(t) \sigma_{z}-\rho_{S}(t)\right],
$$

where $\gamma(t)$ is a time-dependent decoherence rate related to $\Gamma(t)$ as

$$
\gamma(t)=\frac{\dot{\Gamma}(t)}{2}=\frac{2 g^{2}}{N} \sum_{k} \frac{\sin \Omega_{k} t}{\Omega_{k}} .
$$

The dependence of $\gamma(t)$ on $\omega$ has a strong influence on the degree of non-Markovianity of the system dynamics. Indeed while, in general, different non-Markovianity measures are associated with different partitions of the parameter space characterizing the open dynamics of the system, this is not the case for pure decoherence, where the measures introduced in Refs. [35-37], which are relevant instances of informative tools for the characterization of non-Markovianity, lead to the same simple criterion for the occurrence of non-Markovian behavior: pure dephasing of a qubit is Markovian (nonMarkovian) iff $\gamma(t) \geqslant 0[\gamma(t)<0]$. This implies that when $\gamma(t)$ turns negative the information that the environment has acquired about the system flows back and the system "recoheres."

As shown in Fig. 2 a sharp transition in the sign of $\gamma(t)$ occurs for $\omega / J=2$. At such threshold value, $\gamma(t)$ reaches its maximum before turning negative for $\omega / J>2$. A strong deviation from pure monotonic dephasing in the region of non-Markovianity is evident in Fig. 3, where $e^{-\Gamma(t)}$ is plotted against the evolution time and the frequency $\omega$ (in units of $J$ ). Again, a drastic change emerges for $\omega / J>2$ as a backflow of information from the environment to the system occurs, rendering the time evolution of $S$ non-Markovian. Indeed it can be shown that for sufficiently long times decoherence exhibits an exponential decay with decay constant proportional to the spectral density at zero frequency. $\Omega=2 J$ is the threshold

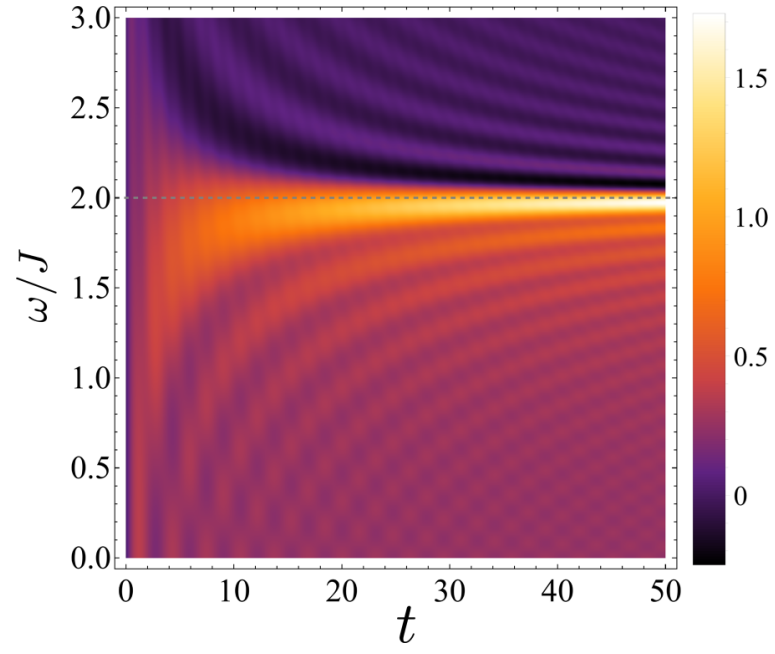

FIG. 2. Time-dependent decay rate $\gamma(t)$ for $\omega \in[0,3]$ (in units of $J)$. A transition between Markovian and non-Markovian dynamics emerges at $\omega=2$, where $\gamma(t)$ reaches its maximum before assuming negative values for $\omega / J>2$.

above which such density is equal to zero. This leads to a deviation from a pure monotonic exponential decay.

\section{QUANTUM DARWINISM AND NON-MARKOVIANITY}

The signature of Darwinism is the presence of a redundancy plateau in the so-called partial information plots (PIP), i.e., the plot of the mutual information $I(S: F)$ shared by the system $S$ and the fragment $F$ of the environment $\mathcal{E}$ accessible by the observers, against the size of $F$ itself. In order to be quantitative, let us consider the reduced joint density operator of the system plus the fragment $\rho_{S F}=\operatorname{Tr}_{R}\left|\Psi_{S \mathcal{E}}\right\rangle\left\langle\Psi_{S \mathcal{E}}\right|$, where the trace is on all the elements of the environment except those belonging to the fragment $F$. We thus call $R$ the part of the array that is traced out, so that $\mathcal{E}=F+R$. We have

$$
I(S: F)=S\left(\rho_{S}\right)+S\left(\rho_{F}\right)-S\left(\rho_{S F}\right),
$$

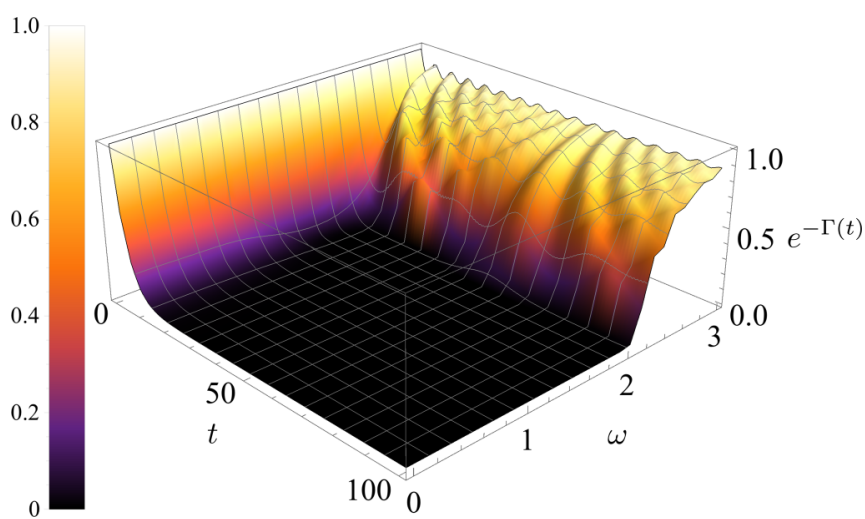

FIG. 3. Decoherence of a single qubit interacting with an environment of $N=201$ oscillators versus the evolution time $t$ and the local-oscillator frequency $\omega$ for $g=0.5$ (all parameters in units of $J$ ). The change in the behavior of the decoherence function $e^{-\Gamma(t)}$ is clearly observed at $\omega / J=2$. 


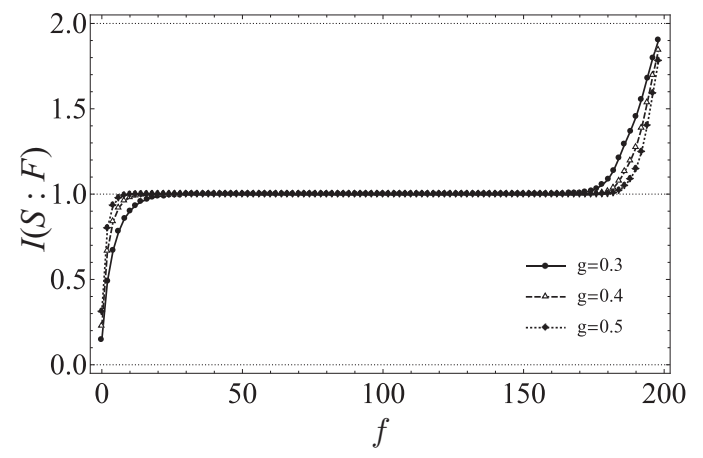

FIG. 4. PIPs showing $I(S: F)$ against the number $f$ of elements in the considered fraction $F$ for an array of $N=201$ oscillators. The three different curves correspond to different values of $g$ (in units of $J$ ), for $\omega / J=0.5$. All the curves show a redundant behavior, which is a characteristic feature of quantum Darwinism. By increasing the coupling constant $g$, the $I(S: F)$ curve shows sharper onset of the plateau, which is a signature of higher degrees of redundancy.

where $S(\rho)=-\operatorname{Tr}(\rho \ln \rho)$ is the von Neumann entropy of state $\rho$.

In the Darwinistic scenario, a PIP exhibits a typical redundant profile [1]: $I(S: F)$ rapidly increases for small values of the dimension $f$ of the fraction being considered and then reaches a plateau at $S\left(\rho_{S}\right)$. This entails the classical plateau: when this amount of information on the state of $S$ is gained, further observations of other subenvironments (i.e., larger values of $f$ ) simply confirm what is already known about the system. The plateau is a characteristic "footprint" of quantum Darwinism: all the fragments contain the same information about the system; the information obtained is objective, since many observers agree about the outcomes.

We now show how the environment of interacting oscillators gains and stores locally redundant information about the system, leading to typical Darwinistic PIPs. To evaluate the quantum mutual information between the system $S$ and growing fractions of the environment it is necessary to go back from the normal modes description to the local-oscillator one. This allows us to follow the dynamics and to evaluate the entropy of the reduced density operator of fragments

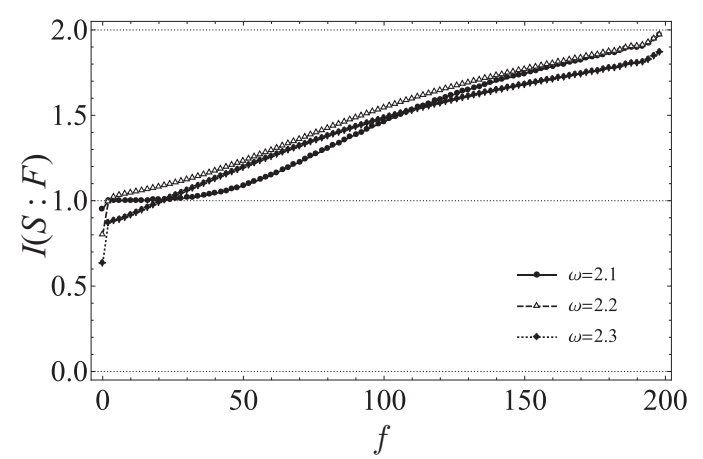

FIG. 5. PIPs similar to the plot reported in Fig. 4. The different curves correspond to different values of $\omega$, all greater than 2 (in units of $J$ ). The redundant encoding of information about $S$ is clearly lost: quantum Darwinism is not emergent, and the amount of the information grows with $f$. consisting of finite sections of the oscillators' array. The time evolution operator in Eq. (4) takes the form of a tensor product of conditional displacement operators each acting on a single bosonic normal mode $k$. Such structure is retained also when the time evolution operator is expressed in terms of local harmonic $j$ oscillators as

$$
\hat{\mathcal{U}}(t)=\bigotimes_{k} \hat{\mathcal{D}}_{c}\left(\beta_{k}\right)=\bigotimes_{j} \hat{\mathcal{D}}_{c}\left(\alpha_{j}\right),
$$

where

$$
\hat{\mathcal{D}}_{c}(\eta)=\exp \left\{\sigma_{z}\left(\eta \hat{\mu}^{\dagger}-\eta^{*} \hat{\mu}\right)\right\}
$$

is the displacement operator with amplitude $\eta=\alpha_{j}=$ $\sum_{k} \beta_{k} e^{i k j} / \sqrt{N}\left(\eta=\beta_{k}\right)$ for $\hat{\mu}=\hat{a}_{j}\left(\hat{\mu}=\hat{b}_{k}\right)$. As already mentioned, we study fragments $F$ of $\mathcal{E}$ consisting of sections centered at the oscillator labeled as $j=0$ and coupled to the system (cf. Fig. 1). However, it is worth mentioning that different choices of fragment arrangements lead to results consistent with what will be discussed in the following.

Without loss of generality, we assume as initial state of the system the balanced superposition $|\psi(0)\rangle_{S}=\left(|0\rangle_{S}+\right.$ $\left.|1\rangle_{S}\right) / \sqrt{2}$ and the harmonic-oscillator array in its ground state. Needless to say, as long as the initial state of the harmonic array is pure, the state of the system-environment compound $\rho_{S \mathcal{E}}(t)$ remains pure at all times and the von Neumann entropy of $\rho_{S F}$ can be quantified through the one of its complementary part $R$. The corresponding mutual information is thus $I(S$ : $F)=S\left(\rho_{S}\right)+S\left(\rho_{F}\right)-S\left(\rho_{R}\right)$.

We start by analyzing the emergence of Darwinism in the Markovian regime, i.e., when $\omega<2 J$. In Fig. 4 we show PIPs for times long enough for the perturbation induced by the coupling with the system to reach the boundaries of the array, $\omega / J=0.5$, and various choices of the coupling constant $g$, as a function of the number $f$ of elements in fragment $F$. The reported results should be taken as typical for the situation studied here. Remarkably, they all exhibit redundant behavior: $I(S: F)$ rapidly increases at small fractions of the environment, then reaches a plateau at $S\left(\rho_{S}\right)=1$. When $f \sim N$, i.e., the entire environment is accessed, $I(S: F)$ again increases sharply and approaches $2 S\left(\rho_{S}\right)$. Therefore, in the Markovian regime the presence of interactions between environmental fragments is not an obstacle to the emergence of quantum Darwinism and we still observe a redundant information encoding about the pointer observable $\hat{\sigma}_{z}^{S}$ in the environment.

Above the threshold $\omega=2 J$, we instead observe the loss of Darwinistic behavior, as shown in Fig. 5. There is no longer a redundancy plateau, and adding fractions of the environment means increasing the amount of information about the system. Darwinism and Markovianity indeed appear as parallel effects: when the time evolution is Markovian, that is $\gamma(t) \geqslant 0$, the interacting environmental fragments still store redundant information about the pointer observable of the system. When the two-level system dynamics acquires non-Markovian features $[\gamma(t)<0]$, quantum Darwinism features disappear, and the quantum mutual information between the system and different fractions of the environment grows approximately linearly. 

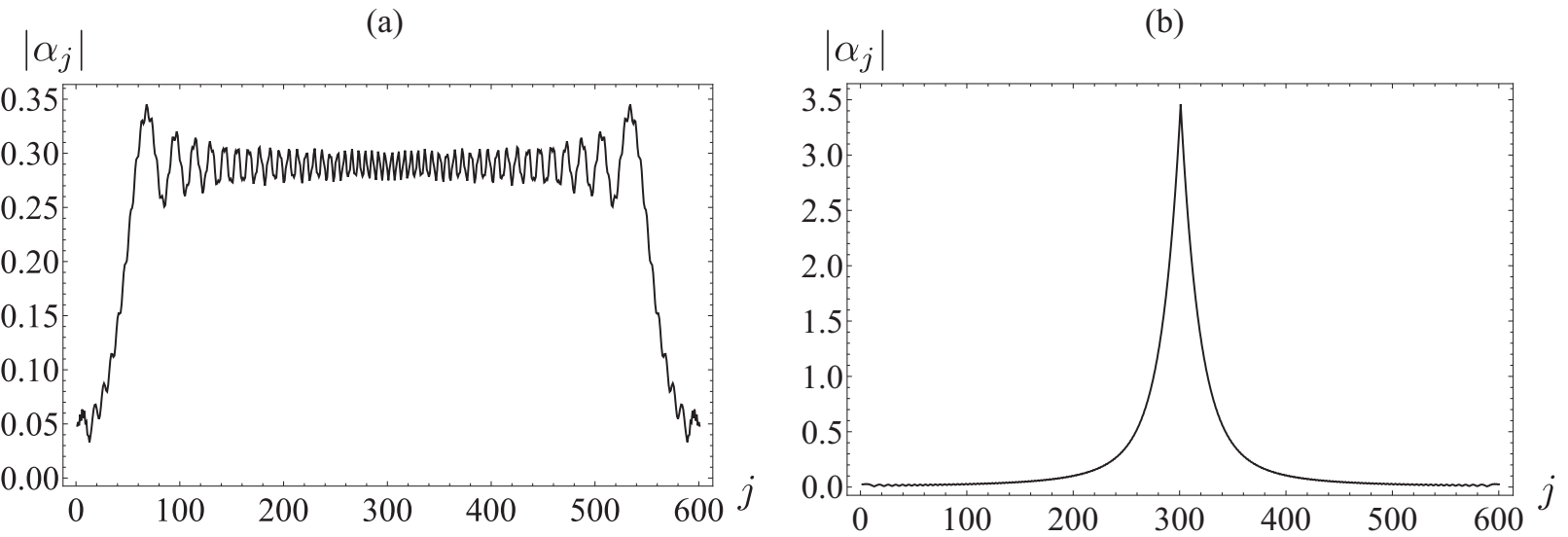

FIG. 6. Panel (a): Amplitude $\left|\alpha_{j}\right|$ of the perturbation induced in the harmonic environment due to the system $S$ for $\omega=1$ (in units of $J$ ). The perturbation spreads through all the environmental subsystems, which in turn determines a Markovian dynamics of the system only. This appears to be correlated to the emergence of quantum Darwinism in light of the redundant encoding of information entailed by the almost uniform spreading of information across $\mathcal{E}$. Panel (b): same as panel (a) but for $\omega / J=2.25$, which sets the emergence of non-Markovian features due to the localization of the perturbation over the array of harmonic oscillators. As we have discussed, this feature is correlated to the breakdown of quantum Darwinism in light of the loss of redundancy in the information encoding process.

\section{INFORMATION PROPAGATION ALONG THE ARRAY}

The correlation between Markovianity and Darwinism in our model can be understood in terms of information flow along the array of interacting harmonic oscillators. Let us first notice that, at $t=0, S$ induces a local perturbation on the array by displacing the central oscillator with $j=0$ from its equilibrium position. Due to the intraenvironment coupling, such local perturbation propagates along the array. In Fig. 6(a) we show the amplitude $\left|\alpha_{j}\right|$ of the perturbation of the array sites again for interaction times long enough for the perturbation to reach the boundaries of the array (in the Markovian regime) and $\omega=J$, a working point that is associated with a Darwinistic and Markovian regime. Panel (b) reports on the results valid for $\omega=2 J$, which puts the system at the onset of non-Darwinistic and non-Markovian conditions. The analysis summarized in Fig. 6 thus shows clearly how the emergence of objective reality as witnessed by a Darwinistic phenomenology appears to be correlated to the features of information spreading across the environment $[49,50]$. Working conditions giving rise to a de facto uniform spread of information across $F$ are associated with Darwinistic trends of $I(S: F)$ in light of the substantial redundance of information encoding about $S$. In turn, a widespread involvement of the set of environmental normal modes is bound to give rise to standard Markovian decoherence of the system's state. The situation becomes strikingly different when only a small part of the environment is affected by the local coupling to $S$. The finiteness of the effective environment gives rise to non-Markovian features as due to information trapping. In turn, Darwinism is prevented by the effective cutoff in the number of subenvironments involved in the open dynamics of $S$. The temporal emergence of the features illustrated above is captured very well by an analysis that moves away from quasi-stationary-state conditions and comprises a range of interaction times. Figure 7 reports on dynamical PIPs contrasting Markovian and non-Markovian regimes. In the Darwinistic-Markovian regime (cf. Fig. 7) as the perturbation (a)

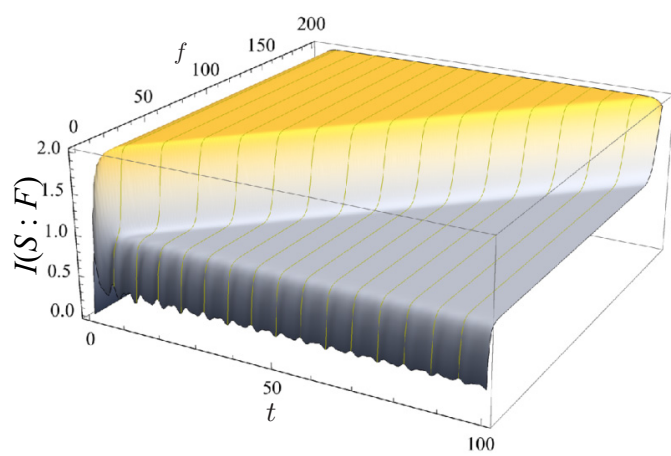

(b)

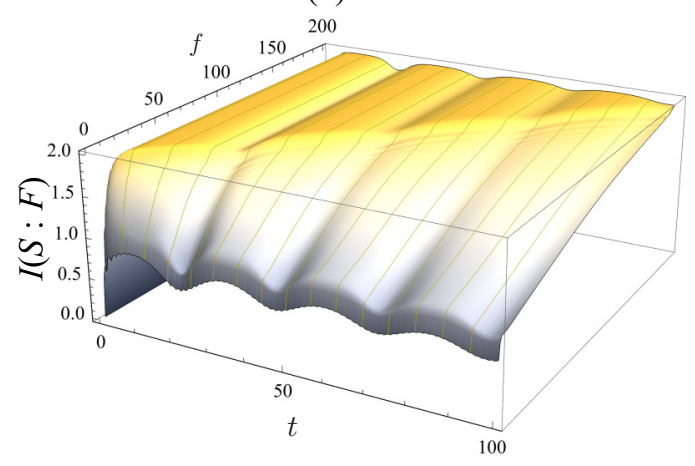

FIG. 7. Panel (a): Temporal PIP for $\omega / J=0.5$ and increasing size $f$ of the environmental fragment $F$ (total number of elements in $\mathcal{E}$ is $N=201$ ). As the time increases, quantum Darwinistic behaviors become apparent. In panel (b), which corresponds to $\omega / J=2.25$, Darwinistic features vanish, as the environment does not store information about $S$ redundantly. As $f$ increases, the amount of the information in the environment grows. 
propagates along the array the redundancy plateau $I(S f)=1$ extends to larger fragment sizes at increasing interaction times before abruptly reaching its maximum value $I(S: F)=2$. When $\omega / J>2$, the encoding of information on $S$ in the environment is no longer redundant but rather increases for larger fragment sizes.

\section{CONCLUSIONS}

Quantum Darwinism has been proposed as a framework for the characterization of the quantum-to-classical transition setting its premises in the objectivity of classical information about a system undergoing open quantum dynamics. Despite its appeal, the Darwinistic phenomenology is only partially understood so far, in particular in relation to the trade-off that its emergence sets with the rich dynamics of an openquantum system. In order to advance our understanding of the phenomenology of quantum Darwinism, we have addressed the case of an exactly solvable interacting environmental model coupled locally to a single two-level system to give rise to a time-dependent dephasing dynamics. Our analysis illustrates a strong correlation between information trapping or spreading, which determines the degree of Markovianity of the ensuing system dynamics, and the emergence of Darwinistic behavior in the amount of information that the environment acquires on the system itself. While providing a clear physical picture of the features of such important characteristics of the system's evolution, our study suggests a possible significant causal link between information spreading and the emergence of objective reality.

\section{ACKNOWLEDGMENTS}

This work was supported by the European Union through project TEQ (Grant No. 766900), the SFI-DfE Investigator Programme QuNaNet (Grant No. 15/IA/2864), and The Leverhulme Trust Research Project Grant. N.M. was partially supported by the Erasmus+ programme and thanks the Centre for Theoretical Atomic, Molecular, and Optical Physics, School of Mathematics and Physics, Queen's University Belfast for hospitality during the early stages of this work.
[1] W. H. Zurek, Nat. Phys. 5, 181 (2009).

[2] W. H. Zurek, Rev. Mod. Phys. 75, 715 (2003).

[3] W. H. Zurek, Phys. Rev. D 24, 1516 (1981).

[4] W. H. Zurek, Phys. Rev. D 26, 1862 (1982).

[5] H. Ollivier, D. Poulin, and W. H. Zurek, Phys. Rev. Lett. 93, 220401 (2004).

[6] R. Blume-Kohout and W. H. Zurek, Found. Phys. 35, 1857 (2005).

[7] R. Blume-Kohout and W. H. Zurek, Phys. Rev. A 73, 062310 (2006).

[8] M. Zwolak, H. T. Quan, and W. H. Zurek, Phys. Rev. Lett. 103, 110402 (2009).

[9] M. Zwolak, H. T. Quan, and W. H. Zurek, Phys. Rev. A 81, 062110 (2010).

[10] C. J. Riedel, W. H. Zurek, and M. Zwolak, New J. Phys. 14, 083010 (2012).

[11] M. Zwolak and W. H. Zurek, Sci. Rep. 3, 1729 (2013).

[12] M. Zwolak, C. J. Riedel, and W. H. Zurek, Phys. Rev. Lett. 112, 140406 (2014).

[13] M. Zwolak, C. J. Riedel, and W. H. Zurek, Sci. Rep. 6, 25277 (2016).

[14] M. Zwolak and W. H. Zurek, Phys. Rev. A 95, 030101(R) (2017).

[15] F. G. S. L. Brandao, M. Piani, and P. Horodecki, Nat. Commun. 6, 7908 (2015)

[16] N. Balaneskovic, Eur. Phys. J. D 69, 232 (2015).

[17] N. Balaneskovic and M. Mendler, Eur. Phys. J. D 70, 177 (2016).

[18] G. Pleasance and B. M. Garraway, Phys. Rev. A 96, 062105 (2017).

[19] F. Galve, R. Zambrini, and S. Maniscalco, Sci. Rep. 6, 19607 (2016).

[20] G. L. Giorgi, F. Galve, and R. Zambrini, Phys. Rev. A 92, 022105 (2015).

[21] T. Unden, D. Louzon, M. Zwolak, W. H. Zurek, and F. Jelezko, arXiv:1809.10456 [quant-ph].
[22] R. Brunner, R. Akis, D. K. Ferry, F. Kuchar, and R. Meisels, Phys. Rev. Lett. 101, 024102 (2008).

[23] A. M. Burke, R. Akis, T. E. Day, G. Speyer, D. K. Ferry, and B. R. Bennett, Phys. Rev. Lett. 104, 176801 (2010).

[24] M. A. Ciampini, G. Pinna, P. Mataloni, and M. Paternostro, Phys. Rev. A 98, 020101(R) (2018).

[25] M.-C. Chen, H.-S. Zhong, Y. Li, D. Wu, X.-L. Wang, L. Li, N.-L. Liu, C.-Y. Lu, and J.-W. Pan, Sci. Bull. 64, 580 (2019).

[26] R. Horodecki, J. K. Korbicz, and P. Horodecki, Phys. Rev. A 91, 032122 (2015).

[27] J. K. Korbicz, P. Horodecki, and R. Horodecki, Phys. Rev. Lett. 112, 120402 (2014).

[28] T. P. Le and A. Olaya-Castro, Phys. Rev. Lett. 122, 010403 (2019).

[29] T. P. Le and A. Olaya-Castro, Phys. Rev. A 98, 032103 (2018).

[30] A. Lampo, J. Tuziemski, M. Lewenstein, and J. K. Korbicz, Phys. Rev. A 96, 012120 (2017).

[31] H.-P. Breuer, J. Phys. B 45, 154001 (2012).

[32] A. Rivas, S. F. Huelga, and M.B. Plenio, Rep. Prog. Phys. 77, 094001 (2014).

[33] H.-P. Breuer, E.-M. Laine, J. Piilo, and B. Vacchini, Rev. Mod. Phys. 88, 021002 (2016).

[34] I. de Vega and D. Alonso, Rev. Mod. Phys. 89, 015001 (2017).

[35] H.-P. Breuer, E.-M. Laine, and J. Piilo, Phys. Rev. Lett. 103, 210401 (2009).

[36] A. Rivas, S. F. Huelga, and M. B. Plenio, Phys. Rev. Lett. 105, 050403 (2010).

[37] S. Lorenzo, F. Plastina, and M. Paternostro, Phys. Rev. A 88, 020102(R) (2013).

[38] E.-M. Laine, J. Piilo, and H.-P. Breuer, Phys. Rev. A 81, 062115 (2010)

[39] T. J. G. Apollaro, C. Di Franco, F. Plastina, and M. Paternostro, Phys. Rev. A 83, 032103 (2011).

[40] P. Rebentrost and A. Aspuru-Guzik, J. Chem. Phys. 134, 101103 (2011). 
[41] P. Haikka, S. McEndoo, G. De Chiara, G. M. Palma, and S. Maniscalco, Phys. Rev. A 84, 031602(R) (2011).

[42] S. Lorenzo, F. Plastina, and M. Paternostro, Phys. Rev. A 84, 032124 (2011).

[43] J.-S. Tang, C.-F. Li, Y.-L. Li, X.-B. Zou, G.-C. Guo, H.-P. Breuer, E.-M. Laine, and J. Piilo, Europhys. Lett. 97, 10002 (2012).

[44] S. Lorenzo, F. Plastina, and M. Paternostro, Phys. Rev. A 87, 022317 (2013).

[45] T. Tufarelli, M. S. Kim, and F. Ciccarello, Phys. Rev. A 90, 012113 (2014).
[46] T. J. G. Apollaro, S. Lorenzo, C. Di Franco, F. Plastina, and M. Paternostro, Phys. Rev. A 90, 012310 (2014).

[47] P. G. , S. Kalle-antti, and E. Artur, Proc. R. Soc. London, Ser. A 452, 567 (1996).

[48] M. A. Cirone, G. D. Chiara, G. M. Palma, and A. Recati, New J. Phys. 11, 103055 (2009).

[49] F. Lombardo, F. Ciccarello, and G. M. Palma, Phys. Rev. A 89, 053826 (2014).

[50] G. Calajo, F. Ciccarello, D. Chang, and P. Rabl, Phys. Rev. A 93, 033833 (2016). 